\title{
Supporting Collaboration through Semantic-based Workflow and Constraint Solving
}

Yun-Heh Chen-Burger', Kit-Ying Hui ${ }^{2}$, Alun D. Preece ${ }^{3}$, Peter M. D. Gray ${ }^{3}$, Austin Tate ${ }^{1}$

${ }^{1}$ School of Informatics, The University of Edinburgh, ${ }^{2}$ School of Computing, The Robert Gordon University, ${ }^{3}$ Computing Science Department, University of Aberdeen

\section{Introduction}

We describe a collaborative problem solving architecture driven by semantic based workflow orchestration and constraint solving. These technologies are based on shared ontologies that allow two systems of very different natures to communicate, perform specialised tasks and achieve common goals. We give an account of our approach for the this case, a system with constraint solving capabilities. We found that systems built with Semantic Web based technologies are useful for collaboration and are easier to add additional specialised capabilities. collaboration and are easier to add additional specialised capabilities.
However, much care must be exercised before correct semantics may be exchanged and collaborations occur smoothly.

Keywords: Virtual Organisation, Constraint Satisfaction, Business Process Modelling, Knowledge Modelling, IDEF3, Ontology, NIST PSL, Semantic Web, Product Synthesis.

\section{Background Technologies}

Formal Business Process Modelling Language (FBPML): FBPML is an extension of a merging of two recognised process modelling
standards: IDEF3 and NIST PSL (the Process Specification Language). FBPML combines these two methods by adapting IDEF3's rich visual and modelling methods and mapping those modelling concepts to the formal semantics and theories of PSL. In addition, based on the design rationale from a business model, FBPML provides comprehensive process execution logic that was
neither included in IDEF3 nor PSL. This business-decision directed neither included in IDEF3 nor PSL. This business-decision directed
execution logic was described and implemented using a First Order execution logic was described and implemented using a First Order
Logic representation. Process descriptions written using FBPML are Logic representation. Process descriptions written using FBPML are
immediately enactable using its workflow system. Data manipulated by the FBPML processes is described in the integrated data language (FBPML-DL) that may be used in its own right. The user may use of FBPML and FBPML-DL are automatically translated to semanticrich OWL-S and OWL descriptions that facilitate interoperability with other systems that use those languages on the Semantic Web. [1] gives other systems that use those 1 more details about this work.
mo

As FBPML and FBPML-DL allow the modeller to express processes and constraints on data, they are also translated to an intermediate language, CIF, to facility the communication with the KRAFT system. Constraint Interchange Format (CIF) is RDF-based and its structure is defined in RDF Schema. One satisfying feature of this constraint interchange format is that the (name) tags used make a clean connectives, and information ant Expressions denoting objects in the data model. Effectively CIF preserves a layer of rich semantic information while providing the processing convenience of RDF [2].

KRAFT (Knowledge Reuse And Fusion/Transformation) is a distributed agent-based information system that emphasizes the use of mobile constraint knowledge to dynamically compose problem instances and tailor them to suit problem solvers. It uses constraints as
a uniform formalism to represent domain-specific knowledge, partially a uniform formalism to represent domain-specific knowledge, partially
solved solutions and intermediate results. The KRAFT architecture solved solutions and intermediate results. The KRAFT architecture
contains "wrappers" that map constraints and data from heterogeneous resources onto a common shared ontology, named integration schema.
When expressed against a KRAFT domain-wide integration schema, objects that can move within a KRAFT-aware agent network [2].

I-X [3] includes a set of tools within a rich systems integration architecture. It visualises, takes decisions and tracks process executions at run-time. Several communication strategies have also been offered to talk with heterogeneous systems of different requirements. These comminication methods are relatively flextible and may be modified a run time. Various work has been proposed and carried out in differen application areas that will seek to create generic approaches (I-Tools) fo
the various types of tasks in which users may engage.

\section{Architecture}

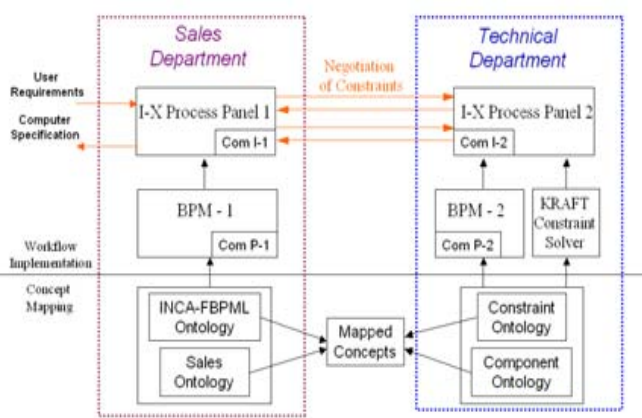

Figure 1. Conceptual Architecture of Workflow Collaboration

Our work is illustrated in a simple example. Consider solving a Personal Computer (PC) configuration problem in a virtual organisation that builds PCs based on customer's individual requirements. Different departments in the organisation are located dispersedly; each may have certain level of overlapping of domain knowledge and operations with another but may also has specific non-overlapping local expertise -- that may be data and/or work procedures related. They need to collaborate with each other to achieve common organisational goals - i.e. to build customer-tailore PCs. In this example, a sales and a technical departments are involved. Each of the sales and technical department has its own internal proces models that govern its operations and may decide to change those operations at run-time, if so desired. Each process model also includes a
set of communication processes that is responsible for the interaction with other internal departments or external organisations.

Figure 1 gives an overview of a conceptual architecture that enables collaborative problem solving using semantic-based workflow lechniques. The horizontal line that goes through the two departments of invides them in two parts has been used to distinguish the two phases conceptual mapping that enables this implementation. Each of the three main technologies involved, FBPML, I-X and KRAFT, is supported by their own underlying methodologies and systems. In this example, two $\mathrm{I}$ $\mathrm{X}$ process panels were used because their facilities suit our
requirements, although other workflow systems offering similar functions may be used instead. I-X process panels manage and monitor workflow execution via process-aware (user) interfaces. The user may choose to alter processes at run-time, if so desired. I-X Process Panels also provide an open communication mechanism to accommodate potentially differen interaction requirements. In this example we have chosen to use KRAFT system as our knowledge agent, due to its rich ability in semantic specialised knowledge aive problem solving power. Other similar and/or appropriate facilities.

As I-X is based on the conceptual framework of $\langle\mathrm{I}-\mathrm{N}-\mathrm{C}-\mathrm{A}\rangle$, FBPML is firstly mapped to $\langle\mathrm{I}-\mathrm{N}-\mathrm{C}-\mathrm{A}\rangle$. This result is indicated in the INCA FBPML ontology in Figure 1 . This enables FBPML business process In addition, the Constraint Ontology that underpins th KRAFT system is mapped to the INCA-FBPML ontology that allows concept mapping between FBPML, I-X and KRAFT systems. During the process of concept mapping, patterns needed to produce correspondence betwee different systems during operations have also been identified and used to form the application context of the communication language. The communication processes within each department, indicated by $\operatorname{Com} P-i$, are a recognised type of process in FBPML and are clearly labelled in models. These communication processes are implemented in separate modules in this example to facilitate the communicatio

Workflow Collaboration with Constraint Solving Capabilities

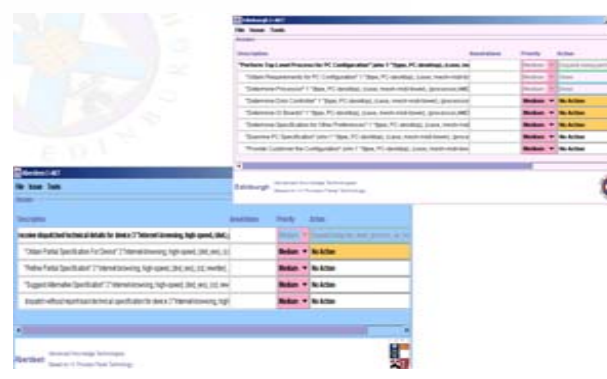

Figure 2. Workflow enactment through I-X process panels

Figure 2 shows the two I-X Process Panels used to instantiate FBPML processes. They assist FBPML dynamic task execution, decision making, communication and collaboration with the KRAFT System. The sales and 'Edinburgh' and 'Aberdeen', panels. In this example, the sales department (Edinburgh) needs to resolve a technical task that is a part of its internal "Selling Customised PC" process. It therefore passes this task to its technical counter-part in Aberdeen. As this problem may be resolved using Constraint Satisfaction Problem (CSP) solving technologies, the relevant processes in Aberdeen decide to use a CSP solver, the KRAFT system. Provided with a problem description generated by the Edinburgh site, the KRAFT system returns found (partial) solutions back to the awaiting process in the Aberdeen I-X panel, which forwards them to
Edinburgh. If a satisfactory solution was not found, the sales department may decide to negotiate through follow-up enquiries.

This experiment demonstrates process and workflow technologies tha were implemented based on an open distributed agent architecture. This scenario mimics real-life situations where centres are often geographically dispersed and each operates at a certain degree of . This distributed structure also allows collaboration throug semantic rich representations enabling an organisation to be agile reaction to rapid changes. Specialised technologies and local autonomy, bridging if collaboration is required. The mapping of the shared ntologies provides a rich and sound foundation towards corre exchange
execution.

\section{Conclusion}

Typically, the knowledge and operations within a virtual organisation

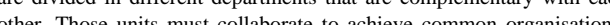
ghels. Distributed workflow systems that loose-couple with specialised knowledge agents provide a suitable framework towards this aim.

In this paper, we demonstrated such a collaboration based on differen technologies: a workflow based (I-X and FBPML) and constrai solving systems (KRAFT). Our work has been successful in the defined ask, but mapping effort was needed in the earlier stages of the project as not all modelling concepts can be easily mapped, so practical solutions must be found. This echoes the complexity of knowledge sharing and interoperability problems between any two or more potentially very arferent but parting ovelapping systens that are known in the

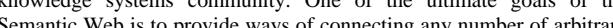
systems to as prove orbitray knowledge. This work is a step towards this goal.

\section{Acknowledgement}

This work is supported under the Advanced Knowledge Technologies Interdisciplinary Research Collaboration, which is sponsored by the UK Engineering and Physical Sciences Research Council under gra by the KRAFT project, funded by the EPSRC and Bitish Tever. Ki Hui's work was performed while at The Aberdeen University for the AKT project. The AKT IRC research partners and sponsors a authorized to reproduce and distribute reprints and on-line copies for heir purposes notwithstanding any copyright annotation hereon.

\section{References}

[1] Yun-Heh Chen-Burger and Jussi Stader. Formal support for adaptive 2003, April 2003.

[2] Kit-Ying Hui, Peter M. D. Gray, Graham J. L. Kemp and Alun D. Preece. Constraints as mobile specifications in e-commerce applications. In $9^{\text {th }}$ IFIP 2.6 Working Conference on Database Semantics,
2001.

[3] Austin Tate, I-X. Technology for intelligent systems. http://www 3] Austin Tate, I-X: Technology for intelligent syst
x.info, AIAI, The University of Edinburgh, 2002 .

\section{Akn}

